\title{
Effect of Moisture Content on the Tensile Strength of Jute Geotextile
}

\author{
Abu Sayed ${ }^{1}$, Md.Zilley Elahi Ibn Salam ${ }^{2}$, Shuvashish Roy $^{3}$ \\ Md.Towhidur Rahman ${ }^{4}$ \\ 1,2,3,4 (Department of Civil Engineering,Bangladesh University of Engineering \&Technology,Bangladesh)
}

\begin{abstract}
Jute geotextile has become a promising and challenging field of interest for the Civil Engineers in the recent years. Use of jute geotextile as an alternative of synthetic geotextile is increasing by a great extent in present days because of its availability and effectiveness in the countries like Bangladesh and India. Use of Jute geotextile instead of synthetic geotextile is cost effective. So a major project is undertaken jointly by India and Bangladesh for the promotion of Jute Geotextile.The effectiveness of Jute Geotextile depends on several properties of jute sample. The main properties are Tensile Strength, Durability, Puncture Resistance etc. In this work, we have done the Grab Tensile strength and wide width Strip Tensile Strength test of different Jute Geotextile sample in various moisture content. The objective of the tests was to observe the variation in Tensile Strength and Elongation of Jute Geotextile sample with the change in moisture content of the sample.From our research we have come to a conclusion that Moisture content has a great impact on the tensile strength and elongation properties of the Jute Geotextiles. Both the Grab tensile strength and wide width strip tensile strength are decreased with the increase in Moisture Content. The elongation is also reduced with the increase in Moisture Content.
\end{abstract}

Keywords: Jute Geotextile, synthetic geotextile, Tensile Strength, moisture content, elongation

\section{Introduction}

Geotextiles are used to filter, stabilize, reinforce, separate, drain and isolate soils in a variety of civil engineering applications. In many cases, geotextiles replace or reduce the need to use natural aggregate construction materials providing both economic and environmental benefits.

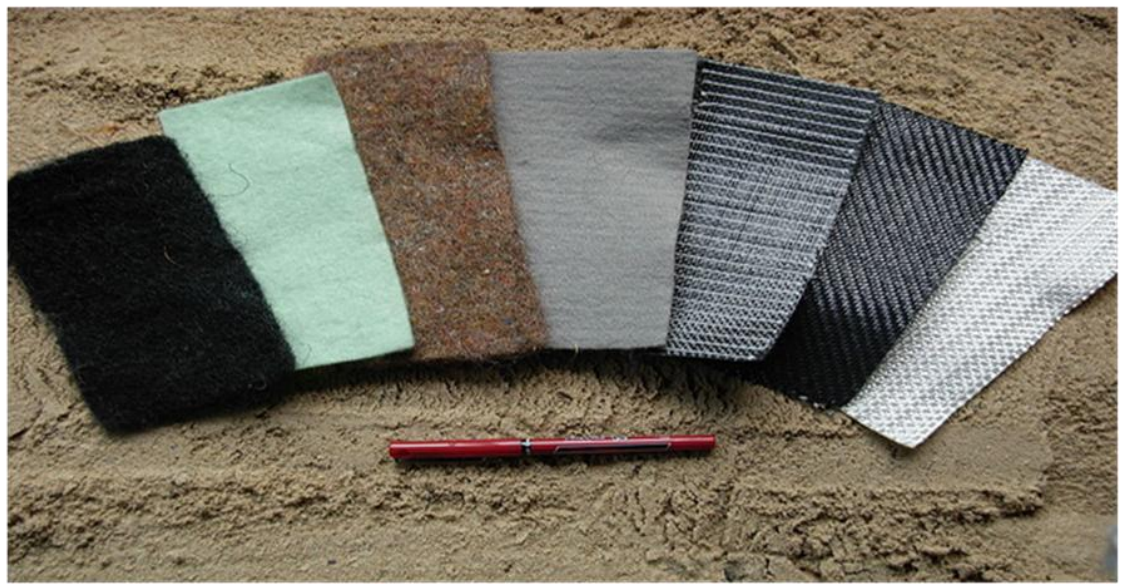

Fig 1: Example of various types of geotextile

Jute geotextile has immense potential both in domestic and international market as it possesses much more advantages than the synthetic fibers.It is used in controlling soil erosion of road side, riverbanks and hill sides. As India is using jute geotextile in the construction of national roads, this could also be used in road construction in Bangladesh[1]. The USA has been using both synthetic and jute geo-textile for road and highways construction.

Jute geotextile is a kind of fiber that addresses geo-technical problems. It is of different kind's coir geotextile, jute geotextile and blend of different fibers. Geotextiles can be effectively used in the management of eroding slopes of roads and railway embankments, mild landslides, prevention of railway track settlement, river embankment and management of solid waste. Presently, some 20,000 tones of jute is being used for geotextiles globally, accounting for about two percent of the market segment. The jute geotextile market has doubled since 1980. 


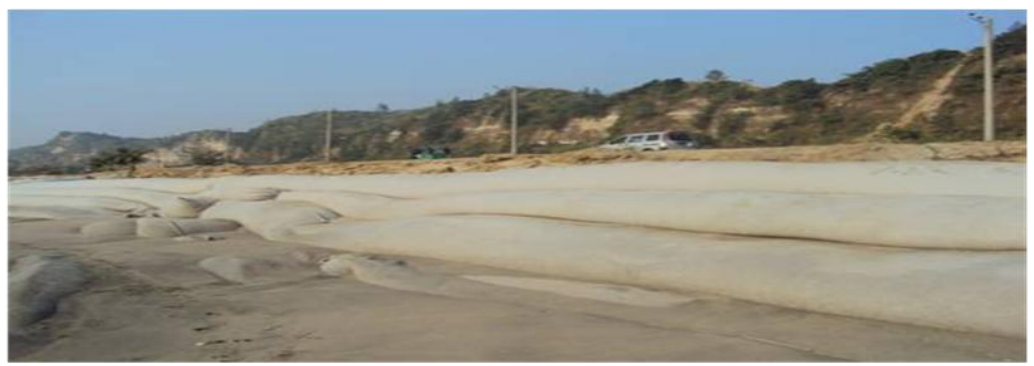

Fig 2: Hill side protection with jute geotextile

Jute geotextile has some advantages. It is biodegradable and it does not spoil the fertility of soil. It is highly hygroscopic and can suck water as well as helps consolidate soil, enhances the flexibility of soil and above all, it is cheaper than the synthetic fibers. Jute geotextiles are textiles used in or near the ground to enhance the ground's characteristics[2]. Applications are usually in the field of civil engineering and environmental engineering and consequently the design of these applications is often closely associated with geo-technical engineering.

\section{Experient}

For this work several tests are done in the laboratory. The tests used are 1)Grab Breaking Load and Elongation Test 2)Wide Width Strip Tensile Strength Test.The ASTM standard methods are followed during the tests.

2.1 Grab Breaking Load and Elongation Test :A tension test in which only a part of the width of the specimen is gripped in the clamps, for fabric testing. It is an index test which provides a procedure for determining the breaking load (grab strength or maximum force to specimen causes rupture ) and elongation (grab elongation or elongation corresponding to the breaking load) of geo-textile, it is useful for quality control test, acceptance test \& determine "effective strength" for a specific fabric structure [3]. And can only be used comparatively between fabrics with very similar structure.

2.2 Wide Width Strip Tensile Strength Test:This test method covers the measurement of tensile strength, \& elongation of the geo-textile, the maximum resistance to deformation developed for a specific material when subjected to tension under existing external load on geo-textile.it also includes direction for the calculation of initial modulus (modulus below tangent point), offset modulus (modulus above tangent point, below proportional limit point), secant modulus (stress and strain ratio for $0 \%$ strain \& $10 \%$ strain) \& breaking toughness.(actual work-to-break per unit surface area of material).the basic distinction between this \& other method of measuring tensile properties of geo-textile is the width of specimen, which is greater than the length in order to minimize the contraction effect (neck down) of jute fabric[4].

\subsection{Determination of Grab tensile strength:}

\subsubsection{Untreated Jute Geotextile (Machine Direction)}

At dry condition:

Sample weight: $13.0 \mathrm{gm}$.

Sample Length: $20.32 \mathrm{~cm}$

Sample Width: $10.16 \mathrm{~cm}$

\begin{tabular}{|l|l|}
\hline Machine Direction & Load, $\mathrm{kg}$ \\
\hline 1 & 125 \\
\hline 2 & 130 \\
\hline 3 & 120 \\
\hline 4 & 125 \\
\hline 5 & 110 \\
\hline
\end{tabular}

Average Load $=(125+130+120+125) / 4$ (here $110 \mathrm{~kg}$ load is ignored $)$

$=125 \mathrm{~kg}$

Tensile Strength $=$ average load/Length of the Sample

$$
\begin{aligned}
& =125 / 10.16 \\
& =12.33 \mathrm{~kg} / \mathrm{cm} \\
& =(12.33 * 9.81 * 100 / 1000) \mathrm{kN} / \mathrm{m} \\
& =12.09 \mathrm{kN} / \mathrm{m}
\end{aligned}
$$

At $15 \%$ moisture content:

Sample weight: $13.1 \mathrm{gm}$.

Sample Length: $20.32 \mathrm{~cm}$

Sample Width: $10.16 \mathrm{~cm}$ 


\begin{tabular}{|l|l|}
\hline Machine Direction & Load, $\mathrm{kg}$ \\
\hline 1 & 120 \\
\hline 2 & 118 \\
\hline 3 & 115 \\
\hline 4 & 114 \\
\hline 5 & 116 \\
\hline
\end{tabular}

Average Load $=(120+118+115+114+116) / 5$

$=116.5 \mathrm{~kg}$

Tensile Strength $=$ average load $/$ Length of the Sample

$$
\begin{aligned}
& =116.5 / 10.16 \\
& =11.47 \mathrm{~kg} / \mathrm{cm} \\
& =(11.47 * 9.81 * 100 / 1000) \mathrm{kN} / \mathrm{m} \\
& =11.25 \mathrm{kN} / \mathrm{m}
\end{aligned}
$$

\subsubsection{Bitumen Treated Jute Geotextile (Machine Direction)}

At dry condition:

Sample weight: $18.0 \mathrm{gm}$.

Sample Length: $20.32 \mathrm{~cm}$

Sample Width: $10.16 \mathrm{~cm}$

\begin{tabular}{|l|l|}
\hline Machine Direction & Load, $\mathrm{kg}$ \\
\hline 1 & 66 \\
\hline 2 & 63 \\
\hline 3 & 62 \\
\hline 4 & 65 \\
\hline 5 & 64 \\
\hline
\end{tabular}

Average Load $=(66+63+62+65+64) / 5$

$$
=64 \mathrm{~kg}
$$

Tensile Strength $=$ average load/Length of the Sample

$$
\begin{aligned}
& =64 / 10.16 \\
& =6.30 \mathrm{~kg} / \mathrm{cm} \\
& =(6.30 * 9.81 * 100 / 1000) \mathrm{kN} / \mathrm{m}
\end{aligned}
$$

$$
=6.18 \mathrm{kN} / \mathrm{m}
$$

At 35\% moisture content:

Sample weight: $18.8 \mathrm{gm}$.

Sample Length: $20.32 \mathrm{~cm}$

Sample Width: $10.16 \mathrm{~cm}$

\begin{tabular}{|l|l|}
\hline Machine Direction & Load, $\mathrm{kg}$ \\
\hline 1 & 21 \\
\hline 2 & 30 \\
\hline 3 & 26 \\
\hline 4 & 28 \\
\hline 5 & 33 \\
\hline
\end{tabular}

Average Load $=(21+30+26+28+33) / 5$

$$
=27.6 \mathrm{~kg}
$$

Tensile Strength $=$ average load/Length of the Sample

$$
\begin{aligned}
& =27.6 / 10.16 \\
& =2.72 \mathrm{~kg} / \mathrm{cm} \\
& =(2.72 * 9.81 * 100 / 1000) \mathrm{kN} / \mathrm{m} \\
& =2.67 \mathrm{kN} / \mathrm{m}
\end{aligned}
$$

2.4 Determination of Wide Width Strip Tensile Strength

2.4.1: Untreated Jute Geotextile (Machine Direction)

At dry condition:

Sample weight: $76.9 \mathrm{gm}$.

Sample Length: $20.32 \mathrm{~cm}$

Sample Width: $20.32 \mathrm{~cm}$

\begin{tabular}{|l|l|}
\hline Machine Direction & Load, $\mathrm{kg}$ \\
\hline 1 & 536 \\
\hline 2 & 540 \\
\hline 3 & 530 \\
\hline 4 & 480 \\
\hline 5 & 580 \\
\hline
\end{tabular}


Average Load $=(536+540+530+580) / 4$ (here $480 \mathrm{~kg}$ load is ignored $)$ $=546.5 \mathrm{~kg}$

Tensile Strength $=$ average load/Length of the Sample

$$
\begin{aligned}
& =546.5 / 20.32 \\
& =26.89 \mathrm{~kg} / \mathrm{cm} \\
& =(26.89 * 9.81 * 100 / 1000) \mathrm{kN} / \mathrm{m} \\
& =26.38 \mathrm{kN} / \mathrm{m}
\end{aligned}
$$

At $35 \%$ moisture content:

Sample weight: $77.30 \mathrm{gm}$

Sample Length: $20.32 \mathrm{~cm}$

Sample Width: $20.32 \mathrm{~cm}$

\begin{tabular}{|l|l|}
\hline Machine Direction & Load, $\mathrm{kg}$ \\
\hline 1 & 465 \\
\hline 2 & 445 \\
\hline 3 & 471 \\
\hline 4 & 452 \\
\hline 5 & 448 \\
\hline
\end{tabular}

Average Load $=(465+445+471+452+448) / 5$

$$
=456.2 \mathrm{~kg}
$$

Tensile Strength $=$ average load/Length of the Sample

$$
\begin{aligned}
& =456.2 / 20.32 \\
& =22.45 \mathrm{~kg} / \mathrm{cm} \\
& =(22.45 * 9.81 * 100 / 1000) \mathrm{kN} / \mathrm{m} \\
& =22.04 \mathrm{kN} / \mathrm{m}
\end{aligned}
$$

\subsubsection{Bitumen treated Jute Geotextile (Machine Direction)}

At dry condition:

Sample weight: $87.9 \mathrm{gm}$.

Sample Length: $20.32 \mathrm{~cm}$

Sample Width: $20.32 \mathrm{~cm}$

\begin{tabular}{|l|l|}
\hline Machine Direction & Load, $\mathrm{kg}$ \\
\hline 1 & 280 \\
\hline 2 & 276 \\
\hline 3 & 269 \\
\hline 4 & 284 \\
\hline 5 & 278 \\
\hline
\end{tabular}

Average Load $=(280+276+269+284+278) / 5$

$$
=277.8 \mathrm{~kg}
$$

Tensile Strength $=$ average load/Length of the Sample

$$
\begin{aligned}
& =277.8 / 20.32 \\
& =13.68 \mathrm{~kg} / \mathrm{cm} \\
& =(13.68 * 9.81 * 100 / 1000) \mathrm{kN} / \mathrm{m} \\
& =13.42 \mathrm{kN} / \mathrm{m}
\end{aligned}
$$

At $30 \%$ moisture content:

Sample weight: $87.9 \mathrm{gm}$.

Sample Length: $20.32 \mathrm{~cm}$

Sample Width: $20.32 \mathrm{~cm}$

\begin{tabular}{|l|l|}
\hline Machine Direction & Load, $\mathrm{kg}$ \\
\hline 1 & 232 \\
\hline 2 & 241 \\
\hline 3 & 245 \\
\hline 4 & 236 \\
\hline 5 & 238 \\
\hline
\end{tabular}

Average Load $=(232+241+245+236+238) / 5$

$$
=238.4 \mathrm{~kg}
$$

Tensile Strength $=$ average load/Length of the Sample

$$
\begin{aligned}
& =238.4 / 20.32 \\
& =11.73 \mathrm{~kg} / \mathrm{cm} \\
& =(11.73 * 9.81 * 100 / 1000) \mathrm{kN} / \mathrm{m} \\
& =11.51 \mathrm{kN} / \mathrm{m}
\end{aligned}
$$




\subsection{Determination of Elongation}

2.5.1 Elongation by Grab Breaking Load Method

\subsubsection{1: Untreated Jute Geotextile (Machine Direction)}

At dry condition

Sample weight: $13.0 \mathrm{gm}$.

Sample Length: $20.32 \mathrm{~cm}$

Sample Width: $10.16 \mathrm{~cm}$

\begin{tabular}{|l|l|}
\hline Machine Direction & Elongation \% \\
\hline 1 & 23 \\
\hline 2 & 22 \\
\hline 3 & 25 \\
\hline 4 & 20 \\
\hline 5 & 15 \\
\hline
\end{tabular}

Average Elongation $=(23+22+25+20+15) / 5$

$$
=21 \%
$$

At 35\% Moisture Content

Sample weight: $13.0 \mathrm{gm}$.

Sample Length: $20.32 \mathrm{~cm}$

Sample Width: $10.16 \mathrm{~cm}$

\begin{tabular}{|l|l|}
\hline Machine Direction & Elongation \% \\
\hline 1 & 16 \\
\hline 2 & 17 \\
\hline 3 & 16 \\
\hline 4 & 15 \\
\hline 5 & 16 \\
\hline
\end{tabular}

AverageElongation $=(16+17+16+15+16) / 5$

$$
=16 \%
$$

\subsubsection{2: Bitumen Treated Jute Geotextile (Machine Direction)}

At dry condition

Sample weight: $18.2 \mathrm{gm}$.

Sample Length: $20.32 \mathrm{~cm}$

Sample Width: $10.16 \mathrm{~cm}$

\begin{tabular}{|l|l|}
\hline Machine Direction & Elongation \% \\
\hline 1 & 11 \\
\hline 2 & 8 \\
\hline 3 & 9 \\
\hline 4 & 8 \\
\hline 5 & 11 \\
\hline
\end{tabular}

Average Elongation $=(11+8+9+8+11) / 5$

$$
=9.4 \%
$$

At 30\% Moisture Content

Sample weight: $18.0 \mathrm{gm}$.

Sample Length: $20.32 \mathrm{~cm}$

Sample Width: $10.16 \mathrm{~cm}$

\begin{tabular}{|l|l|}
\hline Machine Direction & Elongation \% \\
\hline 1 & 9 \\
\hline 2 & 8 \\
\hline 3 & 10 \\
\hline 4 & 9 \\
\hline 5 & 9 \\
\hline
\end{tabular}

AverageElongation $=(9+8+10+9+9) / 5$

$$
=9.4 \%
$$

\subsection{2: Elongation by Wide Width Strip Tensile Strength Method}

\subsubsection{1: Untreated Jute Geotextile (Machine Direction)}

At dry condition

Sample weight: $77.2 \mathrm{gm}$.

Sample Length: $50.8 \mathrm{~cm}$

Sample Width: $20.32 \mathrm{~cm}$ 


\begin{tabular}{|l|l|}
\hline Machine Direction & Elongation \% \\
\hline 1 & 15 \\
\hline 2 & 18 \\
\hline 3 & 17 \\
\hline 4 & 16 \\
\hline 5 & 9 \\
\hline
\end{tabular}

Average Elongation $=(15+18+17+16+9) / 5$

$=15 \%$

At $40 \%$ Moisture Content

Sample weight: $76.9 \mathrm{gm}$.

Sample Length: $50.8 \mathrm{~cm}$

Sample Width: $20.32 \mathrm{~cm}$

\begin{tabular}{|l|l|}
\hline Machine Direction & Elongation \% \\
\hline 1 & 14 \\
\hline 2 & 12 \\
\hline 3 & 13 \\
\hline 4 & 13 \\
\hline 5 & 14 \\
\hline
\end{tabular}

AverageElongation $=(14+12+13+13+14) / 5$

$=13.2 \%$

\subsubsection{2:Bitumen Treated Jute Geotextile (Machine Direction)}

At dry condition

Sample weight: $87.6 \mathrm{gm}$.

Sample Length: $50.8 \mathrm{~cm}$

Sample Width: $20.32 \mathrm{~cm}$

\begin{tabular}{|l|l|}
\hline Machine Direction & Elongation \% \\
\hline 1 & 10 \\
\hline 2 & 11 \\
\hline 3 & 10 \\
\hline 4 & 10 \\
\hline 5 & 10 \\
\hline
\end{tabular}

Average Elongation $=(10+11+10+10+10) / 5$

$=10.2 \%$

At 30\% Moisture Content

Sample weight: $88.2 \mathrm{gm}$.

Sample Length: $50.8 \mathrm{~cm}$

Sample Width: $20.32 \mathrm{~cm}$

\begin{tabular}{|l|l|}
\hline Machine Direction & Elongation \% \\
\hline 1 & 9 \\
\hline 2 & 10 \\
\hline 3 & 11 \\
\hline 4 & 9 \\
\hline 5 & 9 \\
\hline
\end{tabular}

AverageElongation $=(9+10+11+9+9) / 5$

$=9.6 \%$

\section{Results And Discussion}

Grab Tensile Strength and Wide Width Strip Tensile Strength test on various jute geotextile sample are done in the laboratory. Tensile Strength and Elongation are calculated at different moisture content. All the calculations are shown in Chapter - 2. From the Tensile Strength and Elongation value at different moisture content, Tensile Strength vs. Moisture Content and Elongation vs. Moisture Content Graphs are plotted[5].

\subsection{Grab Tensile Strength vs Moisture Content Graph}

\subsection{1: Untreated Jute Geotextile (Machine Direction)}

From Grab Tensile Strength Test on Untreated Jute Geotextile Sample in Machine Direction, the following Data's are found:

\begin{tabular}{|l|l|}
\hline Moisture Content (\%) & Grab Tensile Strength $(\mathrm{kN} / \mathrm{m})$ \\
\hline 0 & 12.09 \\
\hline 15 & 11.25 \\
\hline
\end{tabular}




\begin{tabular}{|l|l|}
\hline 30 & 8.90 \\
\hline 35 & 7.18 \\
\hline 40 & 6.28 \\
\hline
\end{tabular}

From These Data, the Following Graph of Tensile Strength vs Moisture Content is plotted.

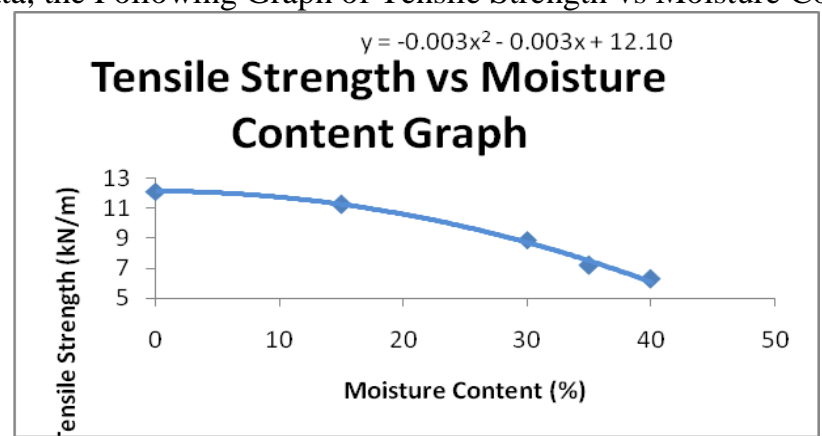

\subsection{2:Bitumen Treated Jute Geotextile (Machine Direction)}

From Grab Tensile Strength Test on Bitumen treated Jute Geotextile Sample in Machine Direction, the following Data's are found:

\begin{tabular}{|l|l|}
\hline Moisture Content (\%) & Grab Tensile Strength $(\mathrm{kN} / \mathrm{m})$ \\
\hline 0 & 6.18 \\
\hline 15 & 5.32 \\
\hline 30 & 3.50 \\
\hline 35 & 2.67 \\
\hline 40 & 1.78 \\
\hline
\end{tabular}

From These Data, the Following Graph of Tensile Strength vs Moisture Content is plotted.

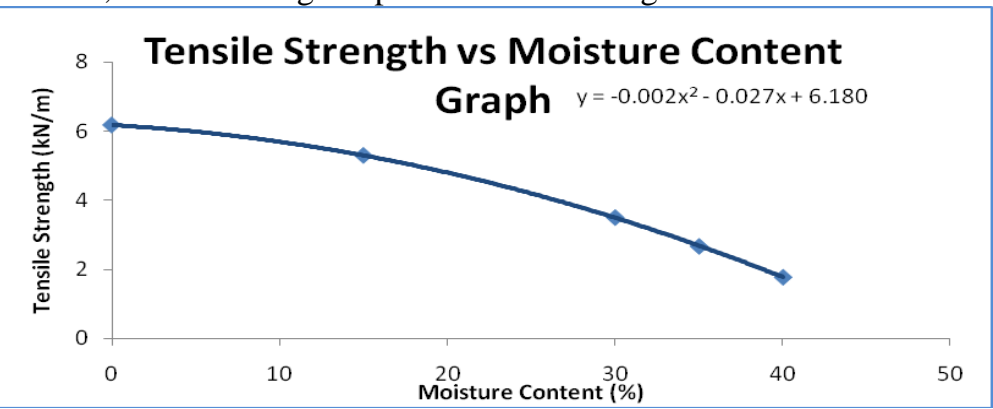

\subsection{Wide Width Strip Tensile Strength vs Moisture Content Graph}

\subsection{1: Untreated JGT (Machine Direction)}

From Wide Width Strip Tensile Strength Test on Untreated Jute Geotextile Sample in Machine Direction, the following Data's are found:

\begin{tabular}{|l|l|}
\hline Moisture Content $(\%)$ & Tensile Strength $(\mathrm{kN} / \mathrm{m})$ \\
\hline 0 & 26.38 \\
\hline 15 & 25.32 \\
\hline 30 & 22.71 \\
\hline 35 & 22.04 \\
\hline 40 & 21.05 \\
\hline
\end{tabular}

From These Data, the Following Graph of Tensile Strength vs Moisture Content is plotted.

\section{Tensile Strength vs Moisture Content Graph}

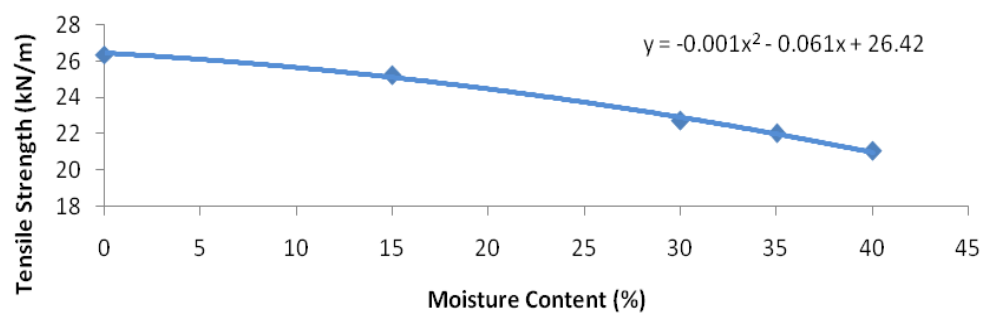




\subsection{Elongation vs Moisture Content Graph (Grab Tensile Strength Test)}

3.3.1: Untreated JGT (Machine Direction)

From Grab Tensile Strength Test on Untreated Jute Geotextile Sample in Machine Direction, the following Data's are found:

\begin{tabular}{|l|l|}
\hline Moisture Content (\%) & Elongation (\%) \\
\hline 0 & 23 \\
\hline 15 & 21 \\
\hline 30 & 20 \\
\hline 35 & 17 \\
\hline 40 & 15 \\
\hline
\end{tabular}

From These Data, the Following Graph of Elongation vs Moisture Content is plotted.

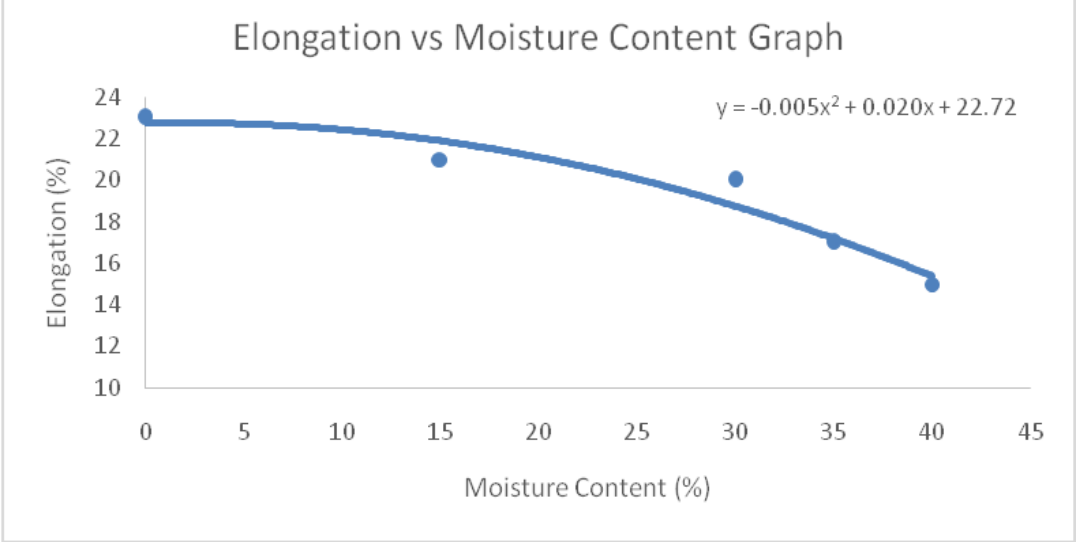

\subsubsection{Bitumen Treated JGT (Machine Direction)}

From Grab Tensile Strength Test on Bitumen treated Jute Geotextile Sample in Machine Direction, the following Data's are found:

\begin{tabular}{|l|l|}
\hline Moisture Content (\%) & Elongation(\%) \\
\hline 0 & 10 \\
\hline 15 & 9 \\
\hline 30 & 9 \\
\hline 35 & 8 \\
\hline 40 & 8 \\
\hline
\end{tabular}

From These Data, the Following Graph of Elongation vs Moisture Content is plotted.

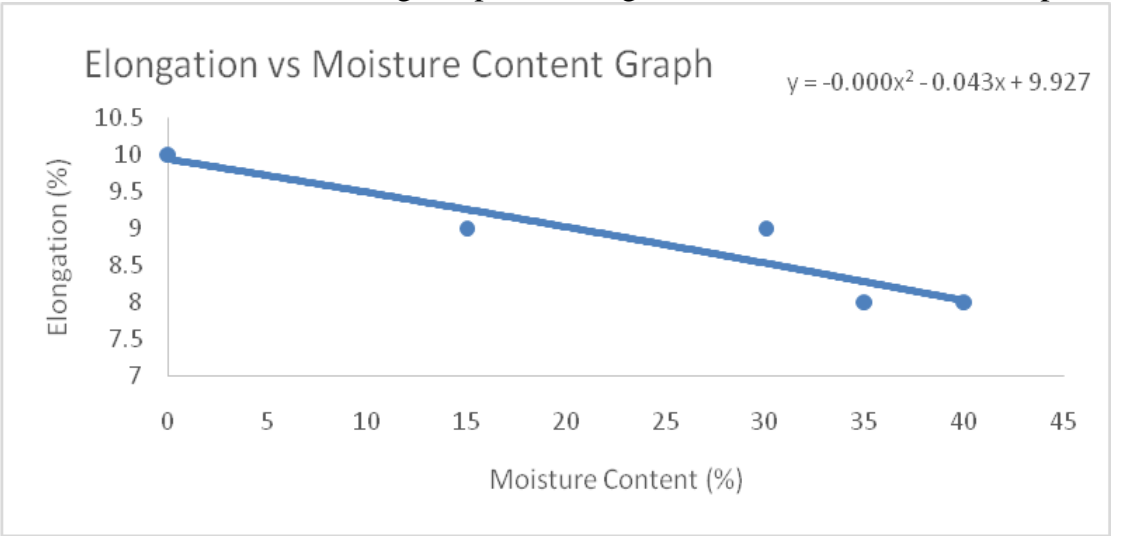

\subsection{Elongation vs Moisture Content Graph (Wide Width Strip Tensile Strength Test)}

\subsection{1: Untreated JGT (Machine Direction)}

From Wide Width Strip Tensile Strength Test on Untreated Jute Geotextile Sample in Machine Direction, the following Data's are found:

\begin{tabular}{|l|l|}
\hline Moisture Content (\%) & Elongation (\%) \\
\hline 0 & 16 \\
\hline 15 & 15 \\
\hline 30 & 14 \\
\hline 35 & 13 \\
\hline 40 & 12 \\
\hline
\end{tabular}


From These Data, the Following Graph of Elongation vs Moisture Content is plotted.

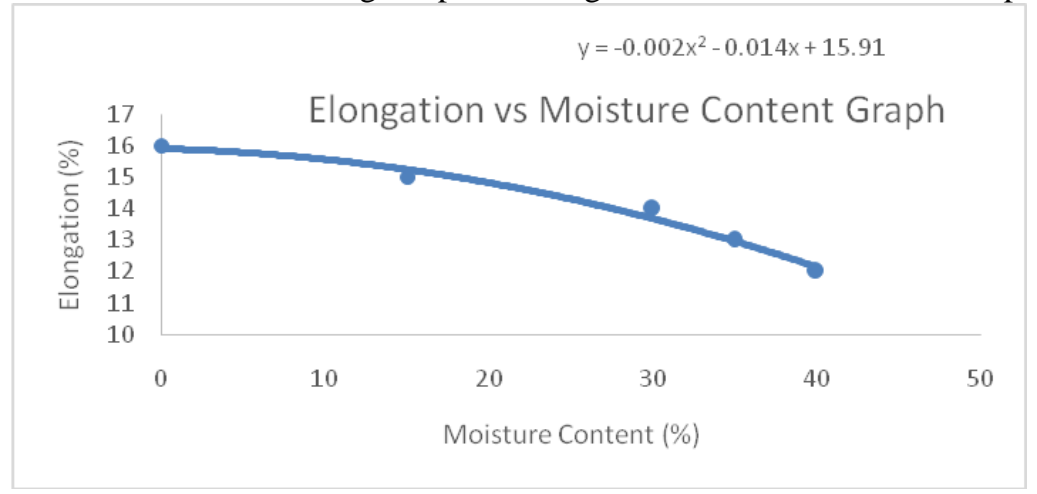

\subsection{2 : Bitumen Treated JGT (Machine Direction)}

From Wide Width Strip Tensile Strength Test on Bitumen treated Jute Geotextile Sample in Machine Direction, the following Data's are found:

\begin{tabular}{|l|l|}
\hline Moisture Content (\%) & Elongation (\%) \\
\hline 0 & 11 \\
\hline 15 & 10 \\
\hline 30 & 10 \\
\hline 35 & 9 \\
\hline 40 & 9 \\
\hline
\end{tabular}

From These Data, the Following Graph of Elongation vs Moisture Content is plotted.

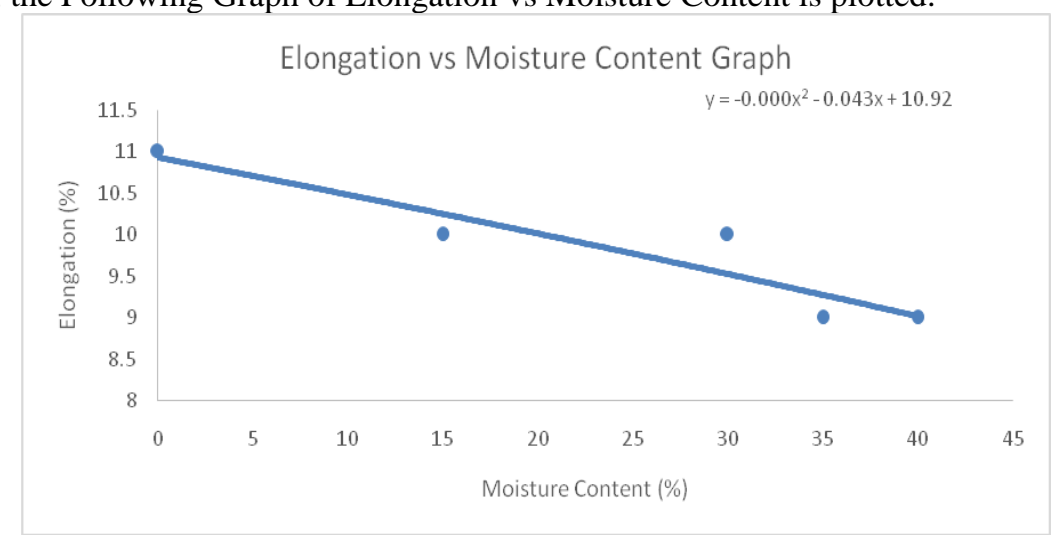

\section{Conclusion}

Jute Geotextiles show different characteristics in different conditions. In case of Tensile Strength, in general, both the Grab Tensile Strength and Wide Width Strip Tensile Strength decreases with the increase in moisture content. The elongation of Jute Geotextiles is also decreases with the increase in moisture content. So we can make a conclusion that the tensile strength and elongation of jute geotextiles depends on its moisture content.Untreated, Bitumen Treated and Latex Treated Jute Geotextiles shows different tensile strength at the same moisture content. So the treatment process also controls the tensile properties of Jute Geotextiles.The Tensile Strength of Jute Geotextiles are also change with the change in Mass per Unit Area (gsm).

\section{References}

[1] Abdullah, A.B.M., (1999). "A hand book on Synthetic geotextiles Particularly Natural Synthetic geotextiles from Jute and other Vegetable Fibres", Bangladesh Jute Research Institute, Dhaka.

[2] Khan A.J. "Technical assessment of Jute Geotextiles for Civil Engineering application" Professor, Department of Civil Engineering, BUET, Dhaka.

[3] ASTM D 4632-91 Standard Test Method for Grab Breaking Load and Elongation of Synthetic geotextiles.

[4] ASTM D 4595-86 (Reapproved 1994) Standard Test Method for Tensile Properties of Synthetic geotextiles by the WideWidth Strip Method.

[5] Mohy, M. A. (2005), Evaluation of properties of JGT and its assessment for short term and long term civil engineering applications. MSc thesis, Department of Civil Engineering, BUET, Dhaka. 